\title{
Gastrointestinal Stromal Tumors: Alexandria University Experience
}

\author{
Maher Soliman ${ }^{1, \odot}$ \\ ${ }^{1}$ Oncology Department, Faculty of Medicine, Alexandria University, \\ Alexandria, Egypt
}

Address for correspondence Maher Soliman, MD, PhD, Oncology Department, Faculty of Medicine, Alexandria University, Alexandria 21526, Egypt (e-mail: maher.soliman@daad-alumni.de).

Asian J Oncol 2021;7:142-148.

\begin{abstract}
Keywords

- GIST

- prognosis

- survival

Introduction Gastrointestinal stromal tumors (GISTs) are the most common mesenchymal tumors of the gastrointestinal tract (GIT) that can arise in any parts of the GIT. The clinical behavior and prognosis of GISTs remain unpredictable. The purpose of this study was to evaluate the clinicopathological features and prognostic factors of GISTs. Methods The medical files of 93 patients with nonmetastatic GIST presented to our hospital were reviewed. The clinical and pathological parameters, treatment, and follow-up data were collected and correlated to survival outcome using univariate and multivariate analyses.

Results The median age of patients was 48.9 years with a slight male predominance. Abdominal pain (39.8\%) was the commonly presenting symptom. About $60 \%$ of GISTs originated from the stomach and $22 \%$ from the small intestine. Tumors stained positive for CD117 in 95.7\%. The median diameter of the tumors was $7 \mathrm{~cm}$. Mitotic counts were $<5 / 50$ high power field in $55.9 \%$ of tumors. About $44 \%$ of patients had high risk tumors. All patients underwent surgery and about $60.2 \%$ of patients received adjuvant imatinib mesylate.

The 5-year disease-free survival (DFS) and overall survival (OS) were 74.5 and $80 \%$, respectively. Margin status, tumor site, tumor size, mitotic counts, and risk score were significantly associated with DFS and OS in both univariate and multivariate analyses. Conclusion Surgery is the mainstay treatment for nonmetastatic GISTs. Tumor size, tumor location, margin status, mitotic count, and risk score were predictive factors for DFS and OS of GISTs.
\end{abstract}

\section{Introduction}

The incidence of gastrointestinal stromal tumors (GISTs) has increased significantly over the last decades especially after the advances in immunohistochemical staining and molecular characterization, comprising the most common mesenchymal tumors of the gastrointestinal (GI) tract. ${ }^{1,2}$ It has been suggested that GISTs arise from the interstitial cells of Cajal or their precursors due to mutations of the c-KIT and less commonly the PDGFRA (platelet-derived growth factor

published online August 16, 2021
DOI https://doi.org/ $10.1055 / \mathrm{s}-0041-1735343$ ISSN 2454-6798 receptor- $\alpha$ ) oncogenes. ${ }^{3}$ Stomach (70\%) and small intestine (20-30\%) are the most common sites of origin of GISTs but they can arise in any parts of the GI tract. ${ }^{1}$ The clinical presentation of GISTs depends on the site and size of the tumor and ranges from asymptomatic small tumor diagnosed incidentally to sizable tumor presenting with GI bleeding, pain, obstruction, or localized mass. ${ }^{4}$

Surgical resection with free safety margins and an intact tumor capsule remains the standard treatment approach for

(C) 2021. Spring Hope Cancer Foundation \& Young Oncologist Group of Asia.

This is an open access article published by Thieme under the terms of the Creative Commons Attribution-NonDerivative-NonCommercial-License, permitting copying and reproduction so long as the original work is given appropriate credit. Contents may not be used for commercial purposes, or adapted, remixed, transformed or built upon. (https://creativecommons.org/licenses/by-nc-nd/4.0/).

Thieme Medical and Scientific Publishers Pvt. Ltd. A-12, 2nd Floor, Sector 2, Noida-201301 UP, India 
primary localized GIST. Unfortunately, the outcome of surgery alone has been insufficient, with up to $50 \%$ of patients developing tumor recurrence within 5 years and eventually dying of disease. The most frequent sites of initial tumor recurrence are the peritoneal surface and the liver., ${ }^{5,6}$

The clinical behavior and prognosis of GISTs vary widely in reported studies. Some tumors show a benign, indolent course, but others follow an aggressive malignant behavior with frequent recurrence and distant metastasis. ${ }^{7}$ GISTs have attracted attention because of their sensitivity to tyrosine kinase inhibitors and imatinib mesylate, an oral form of tyrosine kinase inhibitor, is currently recommended in the adjuvant setting when there is a significant risk of recurrence. ${ }^{8}$ There have been a limited number of publications addressing the clinical features and prognostic factors of GISTs. ${ }^{9-11}$ The purpose of this retrospective study was to evaluate the clinical and pathological features of GISTs and to explore the prognostic factors affecting treatment outcome in patients presented to our tertiary referral hospital.

\section{Methods}

The medical files of all patients with pathologically proven GIST presented to Clinical Oncology Department, Alexandria Main University Hospital during the period from January 2009 to January 2015, were reviewed. Patients with distant metastasis at initial presentation were excluded. Data regarding the demographic features, clinical presentation, investigations, surgery, adjuvant therapy and follow-up data, pattern of recurrence, metastases, and survival was collected. Disease-free survival (DFS) was defined as the time from diagnosis to local or distant recurrence whichever occurred earlier. Overall survival (OS) was calculated from date of diagnosis to date of death. Patients without an event were censored at date of last follow-up.

Tumor size was defined as the largest tumor diameter in any dimension and was stratified as $<5 \mathrm{~cm}, 5$ to $10 \mathrm{~cm}$, or $>10 \mathrm{~cm}$. Margin status was considered as negative (R0) if the entire gross tumor was removed and margins were microscopically tumor-free, while surgery with microscopic infiltrated margin by tumor was considered as R1 and if gross tumor was left was regarded as R2. The risk of aggressive behavior of GISTs was scored according to the National Institutes of Health (NIH) consensus statement of $2001^{12}$ as follows: (a) very low risk: a tumor size $<2 \mathrm{~cm}$ and mitotic count $<5 / 50$ high-power fields (HPF); (b) low risk: a tumor between 2 and $5 \mathrm{~cm}$ and a mitotic count < 5/50 HPF; (c) intermediate risk: a tumor < $5 \mathrm{~cm}$ and mitotic count between 5 and $10 / 50 \mathrm{HPF}$ or a tumor between 5 and $10 \mathrm{~cm}$ and a mitotic count < 5/50 HPF; (d) high risk: a tumor $>10 \mathrm{~cm}$ or mitotic count $>10 / 50 \mathrm{HPF}$ or a tumor $>5 \mathrm{~cm}$ and mitotic count $>$ 5/50 HPF.

\section{Statistical Analysis}

Statistical analyses were performed using SPSS (v. 22) software (SPSS Inc., Chicago, Illinois, United States). Patients were dichotomized into two groups according to the median age ( $\leq$ median vs. $>$ median), sex (male vs. female) and World Health Organization (WHO) performance status (PS) into
( 0 vs. $1-2$ ), tumor location (gastric vs. nongastric) and margin status (negative vs. positive). Patients were categorized according to clinical presentation (pain vs. bleeding vs. anemia vs. others), tumor size ( $<5$ vs. $5-10$ vs. $>10 \mathrm{~cm}$ ), mitotic count ( $<5$ vs. $5-10$ vs. $>10 / 50 \mathrm{HPF}$ ), and NIH risk score (low vs. intermediate vs. high risk).

DFS and OS curves were generated by the Kaplan-Meier method and the two-sided log-rank test was used for comparisons between groups. A multivariate Cox's proportional hazard regression model was used to evaluate prognostic factors and the estimated hazard ratio (HR) with 95\% confidence interval $(\mathrm{CI})$ were calculated. All statistical tests were two sided and $p$-value $\leq 0.05$ was considered statistically significant.

\section{Results}

\section{Clinical Characteristics}

A total of 93 patients with nonmetastatic GIST were recruited. - Table 1 shows the characteristics of patients and tumors. The median age of patients was 48.9 years with (range: 29.5-67.4). There was a slight male predominance with a ratio of 1.8: 1 . Most of patients (81.7\%) had WHO PS of 0 . Abdominal pain was the commonly presenting symptom that was present in $39.8 \%$ of patients. Thirty percent of patients presented by GI bleeding and about $20 \%$ of cases presented by anemia for investigation.

More than half (60.2\%) of GISTs were located in the stomach and about one-quarter (22.6\%) were located in the small intestine. Tumors stained positive for CD117 in 95.7\% and stained positive for CD34 in about $46 \%$ of cases. The median diameter of the tumors was $7 \mathrm{~cm}$ (range: 2.6-30). Thirty-eight patients $(40.9 \%)$ had tumors $<5 \mathrm{~cm}, 24$ patients $(25.8 \%)$ had tumors with size from 5 to $10 \mathrm{~cm}$, and 31 patients (33.3\%) had tumors larger than $10 \mathrm{~cm}$. Mitotic counts were $<5 / 50 \mathrm{HPF}$ in 52 tumors (55.9\%), between 5 and $10 / 50$ HPF in 39 tumors (41.9\%), and > $10 / 50 \mathrm{HPF}$ in only 2 tumors (2.2\%). About one-third of patients (35.5\%) had low risk tumor, 19 patients (20.4\%) had intermediate risk tumors, and 41 patients (44.1\%) had high risk tumors according to NIH risk score.

\section{Treatment}

All patients underwent surgery and complete surgical resection (R0) was achieved in 80 patients (86\%) and 13 patients had positive margin (R1-R2). Three out of 13 patients with incomplete surgery presented by acute abdomen consistent with perforated appendicitis and on exploration it was found to be localized tumor rupture with acute localized peritonitis (R2). Another four patients presented with huge mesenteric GISTs infiltrating the retroperitoneal tissues where cytoreductive surgery was feasible (R2) and the remaining six patients underwent complete surgery but postoperative pathology revealed microscopically positive margin (R1). A total of 56 patients (60.2\%) including the 13 patients with positive margins received adjuvant imatinib mesylate $400 \mathrm{mg}$ daily (27 patients for 12 months, 2 patients for 18 months, 4 patients for 24 months, and 23 patients for 36 months). 
Table 1 Characteristics of patients and tumors

\begin{tabular}{|c|c|c|}
\hline Variable & $n$ & $\%$ \\
\hline Age (median) & 48.9 & $29.5-67.4$ \\
\hline Size (median) & 7 & $2.6-30$ \\
\hline \multicolumn{3}{|l|}{ Gender } \\
\hline Males/females & $60 / 33$ & $64.5 / 35.5$ \\
\hline \multicolumn{3}{|l|}{ PS } \\
\hline $0 / 1-2$ & $76 / 17$ & $81.7 / 18.3$ \\
\hline \multicolumn{3}{|l|}{ Presentation } \\
\hline Pain/ bleeding/ anemia/ others & $37 / 28 / 18 / 10$ & $39.8 / 30.1 / 19.4 / 10.8$ \\
\hline \multicolumn{3}{|l|}{ Site } \\
\hline Gastric & 56 & 60.2 \\
\hline Nongastric (SI/ Mes./ Col./ Rect./ App.) & $37(21 / 6 / 5 / 2 / 3)$ & $39.8(22.6 / 6.5 / 5.4 / 2.2 / 3.2)$ \\
\hline \multicolumn{3}{|l|}{ Size $(\mathrm{cm})$} \\
\hline$<5 / 5-10 />10$ & $38 / 24 / 31$ & $40.9 / 25.8 / 33.3$ \\
\hline \multicolumn{3}{|l|}{ CD117 } \\
\hline Positive/negative & $89 / 4$ & $95.7 / 4.3$ \\
\hline \multicolumn{3}{|l|}{ CD34 } \\
\hline Positive/negative/ unknown & $43 / 19 / 31$ & $46.2 / 20.4 / 33.3$ \\
\hline \multicolumn{3}{|l|}{ Mitotic count (/50HPF) } \\
\hline$<5 / 5-10 />10$ & $52 / 39 / 2$ & $55.9 / 41.9 / 2.2$ \\
\hline \multicolumn{3}{|l|}{ NIH risk score } \\
\hline Low/ intermediate/ high & $33 / 19 / 41$ & $35.5 / 20.4 / 44.1$ \\
\hline \multicolumn{3}{|l|}{ Margin status } \\
\hline Negative/ positive & $80 / 13$ & $86.0 / 14.0$ \\
\hline \multicolumn{3}{|l|}{ Adjuvant IM } \\
\hline No/yes & $37 / 56$ & $39.8 / 60.2$ \\
\hline
\end{tabular}

Abbreviations: App, appendix; Col, colon; HPF, high-power field; IM, imatinib mesylate; Mes, mesentery; NIH, National Institutes of Health; Rect, rectum; SI, small intestine.

\section{Disease-Free Survival}

The median duration of follow-up was 51.5 months (range: 36.5-103.8). There was local recurrence alone in 14 patients, distant metastasis alone in 3 patients, and both in 6 patients. The 5-year DFS was 74.5\% (mean DFS: $79.8 \pm 3$ months). On univariate analysis ( $\bullet$ Fig. 1 ), age $(p=0.893, \neg$ Fig. 1A), gender $(p=0.731$, -Fig. 1B $)$, PS $(p=0.645,-$ Fig. 1C), and clinical presentation ( $p=0.910,-$ Fig. 1D) had no significant impact on DFS. However, margin status ( $p=0.000,-$ Fig. 1E), tumor site $(p=0.000,-$ Fig. 1F), tumor size $(p=0.000$, - Fig. 1G), mitotic counts $(p=0.000,-$ Fig. $1 \mathbf{H})$, and risk score $(p=0.000$, -Fig. 1I) were significantly associated with DFS.

On multivariate analysis ( - Table 2), positive margin (HR: 4.373, 95\% CI: 1.536-12.448, $p=0.006$ ), nongastric location (HR: 8.173, 95\% CI: $1.888-35.382, p=0.005$ ), large tumor size (HR: $1.146,95 \% \mathrm{CI}: 1.047-1.255, p=0.003$ ), high mitotic count (HR: $1.367,95 \% \mathrm{CI}: 1.130-1.653, p=0.001$ ), and high NIH risk score (HR: 5.566, 95\% CI: 1.227-25.250, $p=0.026$ ) were significant predictive factors of unfavorable DFS.

\section{Overall Survival}

Twenty-one patients died during the follow-up period. The 5 -year OS was $80 \%$ (mean OS: $83 \pm 3.6$ months). On univariate analysis ( $\boldsymbol{- F i g . 2}$ ), OS was not significantly associated with age $(p=0.786,-$ Fig. 2A $)$, gender $(p=0.899,-$ Fig. 2B $)$, PS ( $p=0.485,-$ Fig. 2C), and clinical presentation $(p=0.803$, -Fig. 2D). However, the OS was significantly correlated to margin status ( $p=0.000,-$ Fig. $\mathbf{2 E}$ ), tumor site $(p=0.000$, -Fig. 2F), tumor size ( $p=0.000,-$ Fig. 2G), mitotic counts $(p=0.000,-$ Fig. $2 \mathrm{H})$, and risk score $(p=0.000,-$ Fig. $2 \mathbf{I})$.

On multivariate analysis (- Table 2), positive margin (HR: 5.135, 95\% CI: $1.756-15.015, p=0.003$ ), nongastric GISTs (HR: 6.346, 95\% CI: $1.218-33.057, p=0.028$ ), larger tumor size (HR: 1.196, 95\% CI: $1.069-1.338, p=0.002$ ), and NIH risk score (HR: 8.745, 95\% CI: 1.740-43.944, $p=0.008$ ) were significant prognostic factors for poor OS. However, mitotic counts were marginally significantly (HR: $1.235,95 \% \mathrm{CI}: 0.994-1.533, p$ $=0.056$ ) associated with OS. 

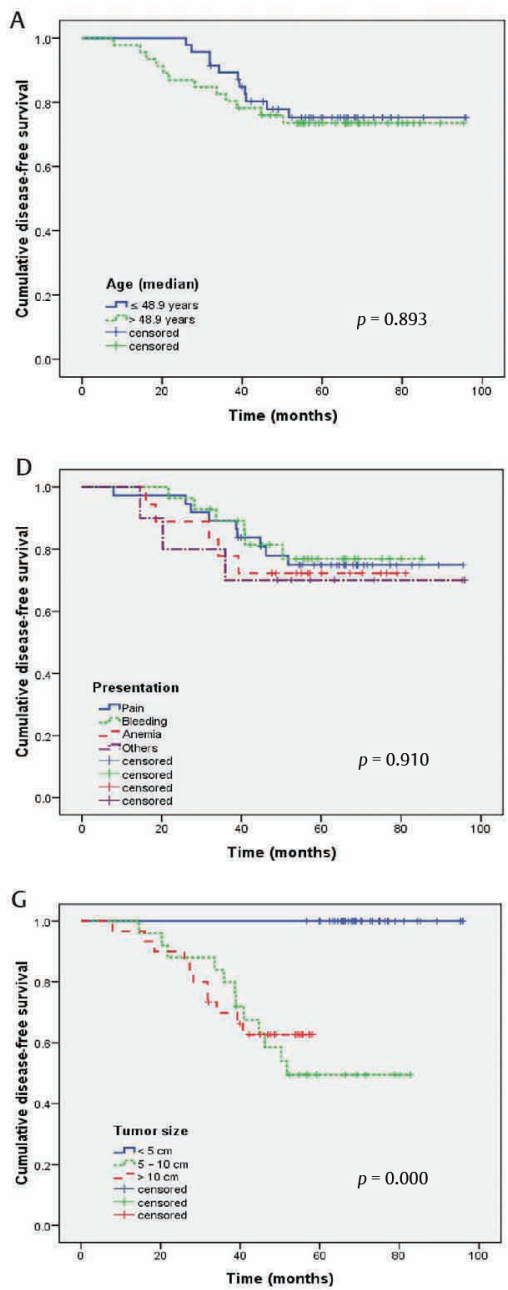
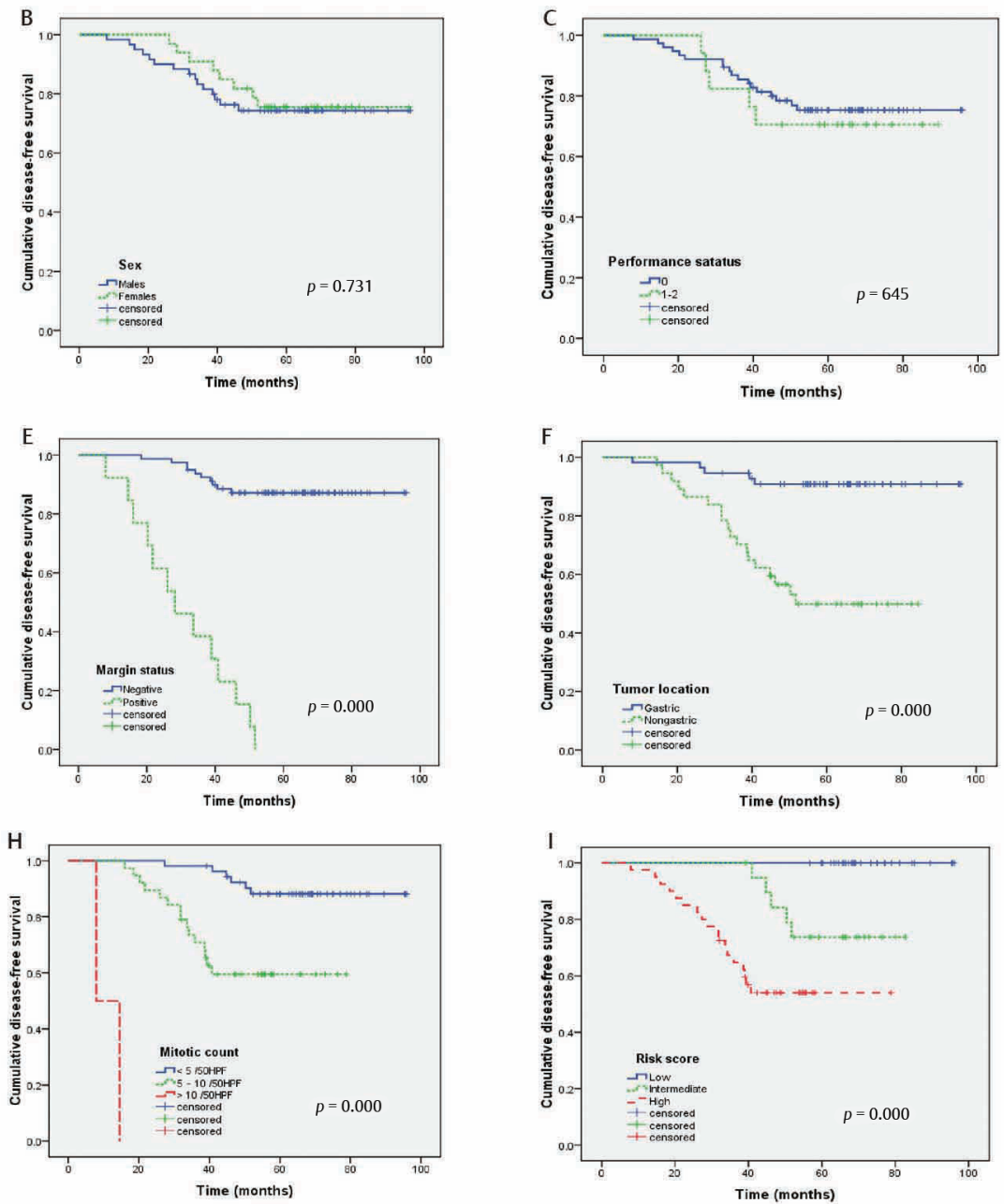

Fig. 1 Disease-free survival for 93 patients with nonmetastatic gastrointestinal stromal tumors according to age (A), sex (B), performance status $(\mathbf{C})$, presentation $(\mathbf{D})$, margin status $(\mathbf{E})$, tumor location $(\mathbf{F})$, tumor size $(\mathbf{G})$, mitotic count $(\mathbf{H})$, and risk score (I).

\section{Discussion}

It had been reported that the incidence of GISTs is on the fast rise, probably due to the increasing awareness of disease in the last decades. However, GISTs remain rather rare tumors with unpredictable biological behavior and variable clinical course. It is generally accepted that there are no perfect criteria that can exactly predict the prognosis of GIST, and there must be some unrevealed room for improving and adjusting the present predictive parameters.

The median age of our patients was one decade younger ( 48.9 years) than that reported in literature by Kim et $\mathrm{al}^{13}$ who found mean age of 60.8 years and Wang et $\mathrm{al}^{14}$ who evaluated 497 GIST patients with a median age of 60 years. However, it is similar to previous Egyptian study by El-Zohairy et $\mathrm{al}^{15}$ who reported the mean age of patients was 52.8 years. Similarly, Call et al ${ }^{16}$ and Hong et $\mathrm{al}^{17}$ found a median age of 52 years old.

Our data revealed that there was a slight male predominance. Multiple previous reports agree with us. Miettinen et $\mathrm{al}^{4}$ analyzed 1,765 patients with GISTs and found a slight male predominance (55\%). Wang et $\mathrm{al}^{14}$ reported $55.9 \%$ of patients were men. Tran et al ${ }^{18}$ reviewed 1,458 cases of GISTs and found $54 \%$ were men. However, there was no clear sex predilection in most published studies ${ }^{19-21}$ and female predominance was demonstrated in three Korean studies. ${ }^{13,22,23}$

Most GISTs present by nonspecific symptom, increasing the difficulty of early diagnosis and treatment. In our study, consistent with the literature, the most frequent complaints were abdominal pain and GI bleeding. The same was reported by Lin et $\mathrm{al}^{24}$ and Wang et al. ${ }^{14}$ Our data illustrated an incidence of GI bleeding as high as $30.1 \%$. As GISTs usually originate from the muscle layer and may grow into the submucosa and lamina propria, leading to mucosal ulceration and hemorrhage. Similarly, Huang et $\mathrm{a}^{25}$ showed the incidence of GI bleeding in GIST patients was 30.9\% and Wang et al ${ }^{14}$ reported bleeding in $28.6 \%$ of cases.

The distribution of sites of GISTs in the current study was similar to that published in the literature. ${ }^{20,21,24}$ Wang et $\mathrm{al}^{14}$ reported that stomach and small bowel were the most common sites of GISTs (59.0 and 22.5\%, respectively). Lin et $\mathrm{a}^{24}$ showed that $54 \%$ of tumors located in the stomach and $36 \%$ in the small intestine.

The median diameter of the tumors in our cohort was $7 \mathrm{~cm}$ and about $40 \%$ of patients presented by small tumors $<5 \mathrm{~cm}$. Our findings are supported by Lin et $\mathrm{al}^{24}$ who reported 
Table 2 Multivariate analysis of disease-free survival and overall survival

\begin{tabular}{|l|l|l|l|l|l|l|}
\hline & \multicolumn{3}{|c|}{ Disease-free survival } & \multicolumn{3}{c|}{ Overall survival } \\
\hline Variables & $p$-Value & HR & $95 \% \mathrm{Cl}$ for HR & $p$-Value & HR & $95 \%$ CI for HR \\
\hline Margin status & 0.006 & 4.373 & $1.536-12.448$ & 0.003 & 5.135 & $1.756-15.015$ \\
\hline Site & 0.005 & 8.173 & $1.888-35.382$ & 0.028 & 6.346 & $1.218-33.057$ \\
\hline Size & 0.003 & 1.146 & $1.047-1.255$ & 0.002 & 1.196 & $1.069-1.338$ \\
\hline Mitotic count & 0.001 & 1.367 & $1.130-1.653$ & 0.056 & 1.235 & $0.994-1.533$ \\
\hline NIH risk score & 0.026 & 5.566 & $1.227-25.250$ & 0.008 & 8.745 & $1.740-43.944$ \\
\hline
\end{tabular}

Abbreviations: $\mathrm{Cl}$, confidence interval; $\mathrm{HR}$; hazard ratio; $\mathrm{NIH}$, National Institutes of Health.

A
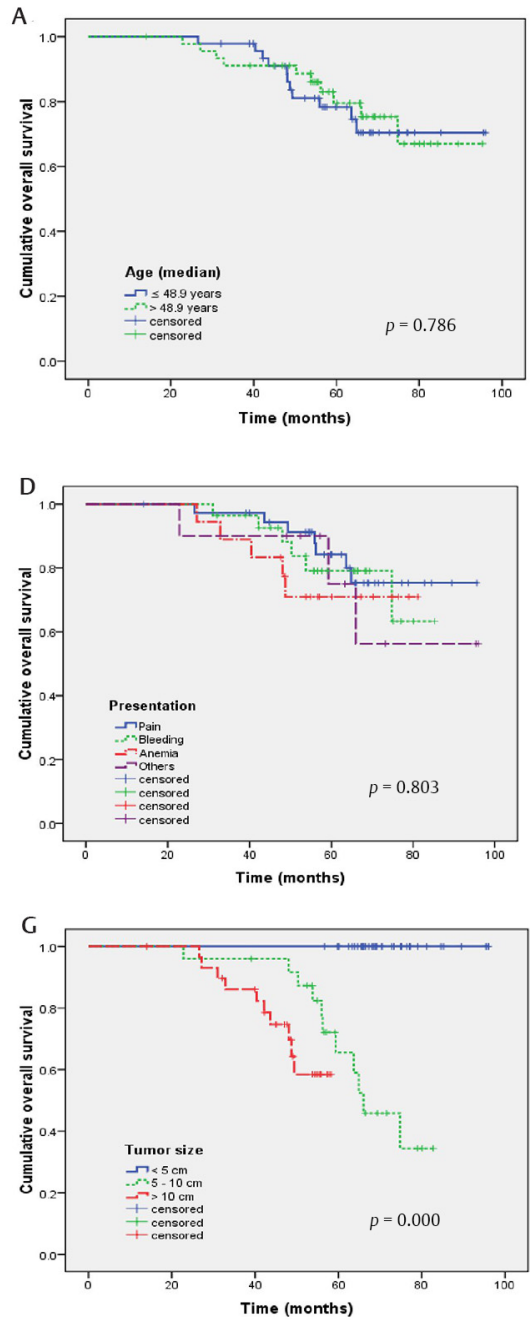
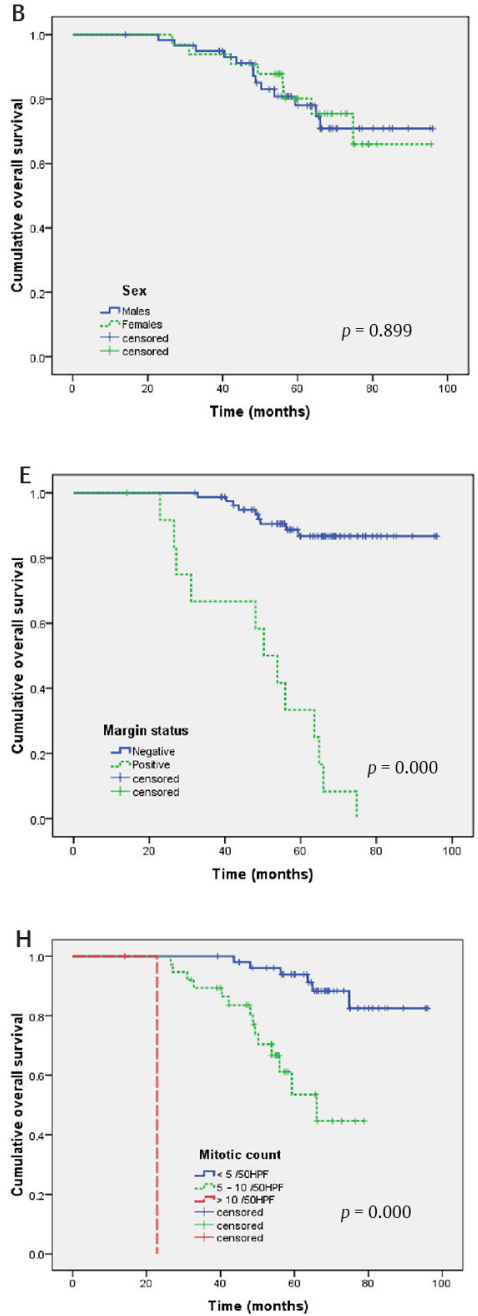
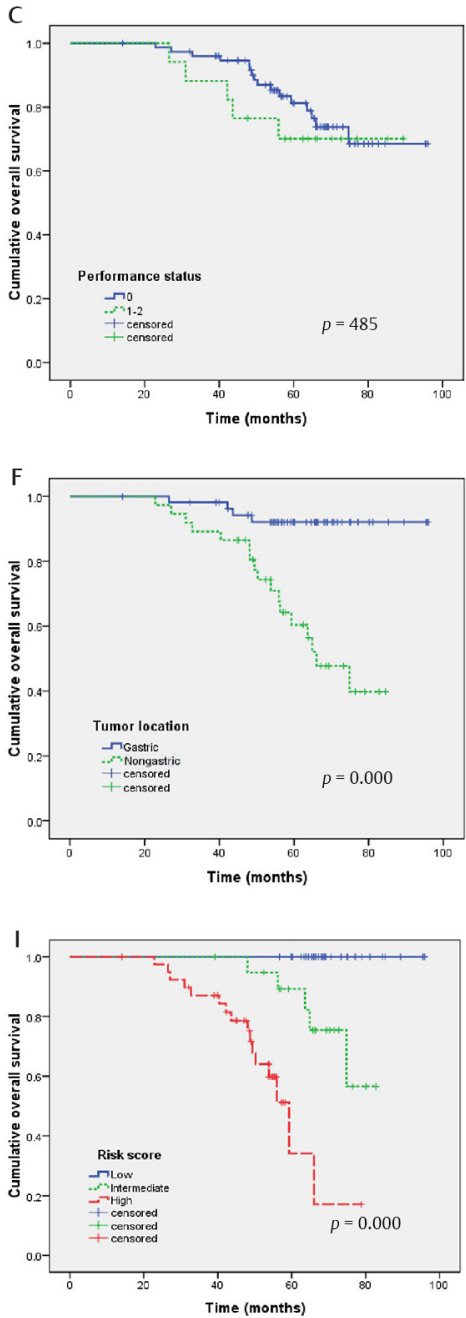

Fig. 2 Overall survival for 93 patients with nonmetastatic gastrointestinal stromal tumors according to age (A), sex (B), performance status $(\mathbf{C})$, presentation $(\mathbf{D})$, margin status $(\mathbf{E})$, tumor location $(\mathbf{F})$, tumor size $(\mathbf{G})$, mitotic count $(\mathbf{H})$, and risk score $(\mathbf{I})$.

that $43 \%$ of patients had tumors $<5 \mathrm{~cm}$ in size. Jumniensuk and Charoenpitakchai ${ }^{26}$ found the median size of GIST was $6.8 \mathrm{~cm}$. However, Kim et $\mathrm{al}^{27}$ evaluated 1,057 GISTs and found median tumor size was $4 \mathrm{~cm}$. Park et $\mathrm{al}^{28}$ reported the median tumor size was $3.1 \mathrm{~cm}$. The median tumor size was smaller in the Eastern studies conducted in Korea and Japan due to improved nationwide surveillance for gastric cancer leading to earlier detection.

Lin et $\mathrm{al}^{24}$ reported that CD117 was positive in $89 \%$ of tissue samples and there were $72.8 \%$ positive for CD34. Consistently, in the present study, tumors stained positive for CD117 in $95.7 \%$. However, CD34 stained positive in only $46 \%$ of cases as CD34 was not routinely done in our hospital due to financial reasons and CD34 staining was unknown in about one third of cases.

In the current study, about $44 \%$ of patients had high risk tumors and $20 \%$ had intermediate risk tumors. Compared to published literature, very low risk group was not represented in our data likely due to lack of screening program and late diagnosis. Wang et $\mathrm{al}^{14}$ reported the distribution of very low-, 
low-, intermediate-, and high-risk groups was 8.0, 36.4, 15.7, and $39.8 \%$, respectively. Huang et $\mathrm{al}^{25}$ reported that the risk categories of 333 GIST patients were very low risk (4.8\%), low risk (18.9\%), intermediate risk (17.7\%), and high risk (47.7\%).

In the present study, the 5-year DFS was $74.5 \%$ and the 5 -year OS was $80 \%$. Consistent with our study, Wang et $\mathrm{al}^{14}$ reported the 5-year DFS and OS of 401 patients with operable GIST were 77.4 and $84.8 \%$, respectively. Huang et $\mathrm{al}^{25}$ reported that the 5 -year DFS was $70.9 \%$. Call et a ${ }^{16}$ found that 5-year OS for 1,215 patients with GIST was $79 \%$.

Different prognostic factors identified previously were investigated in the current study. On univariate and multivariate analyses, margin status, tumor site, tumor size, mitotic counts, and NIH risk score were significantly associated with both DFS and OS. Our results regarding these factors reached a general agreement with prior reports, showing that larger tumor size, high mitotic counts, and nongastric locations and positive resection margin were steadily indicative of unfavorable DFS and OS for patients with GIST. Favorable prognosis of gastric GISTs might be related to early presentation, diagnosis, and treatment. Mitotic count is among the important prognostic factors in GIST and listed in NIH criteria. However, it is unreliable and subjective index and the number of mitoses detected depends on the tissue fixation time, the magnification of the field under the microscope, and the experience of the examiner.

This is in concordance with prior studies and the data of others. Several studies indicated that tumor size, mitotic count, and primary tumor location were significant and reliable prognostic factors for OS. Lin et $\mathrm{a}^{24}$ found that tumor size, mitotic count, and resectability correlated significantly with tumor recurrence and survival. Wang et $\mathrm{al}^{14}$ demonstrated that nongastric origin, larger tumor size, higher mitotic rate, higher risk grade, adjacent organ involvement contributed to poorer outcome (lower DFS and OS) in univariate analysis.

Wang et $\mathrm{a}^{29}$ evaluated the prognostic factors for GISTs in 84 patients after R0 resection and demonstrated on univariate analysis that GI bleeding, nongastric tumor location, large tumor size, high mitotic count were poor prognostic indicators for DFS.

Our findings confirmed that complete resection of the tumor with R0 is one of the most important prognostic factors for OS. Similarly, DeMatteo et $\mathrm{al}^{30}$ found on univariate analysis that tumor size and complete resection of gross disease were significant predictors of survival. Lin et $\mathrm{al}^{24}$ found that complete resection of the tumor is one of the most important factors related to survival.

This study has certain limitations including being a retrospective study with a relatively smaller sample size, a large proportion of censored cases and lack of data about other risk factors like tumor rupture and serosal invasion. However, our study was conducted in a single tertiary hospital including homogenous population cohort with long-term follow-up period and uniform treatment. Despite these caveats, it appears that our results can contribute to the growing body of evidence about the prognostic factors in patients with GISTs.

In conclusion, surgical resection with negative margin is the mainstay treatment for nonmetastatic GISTs with additional imatinib mesylate as adjuvant therapy for intermediate and high-risk groups. Large tumor size, nongastric tumor location, positive tumor margin, high mitotic count, and high NIH risk score are predictive for unfavorable DFS and OS of GISTs.

\section{Conflict of Interest}

None declared.

\section{References}

1 Steigen SE, Eide TJ. Trends in incidence and survival of mesenchymal neoplasm of the digestive tract within a defined population of northern Norway. APMIS 2006;114(3):192-200

2 Nilsson B, Bümming P, Meis-Kindblom JM, et al. Gastrointestinal stromal tumors: the incidence, prevalence, clinical course, and prognostication in the preimatinib mesylate era-a population-based study in western Sweden. Cancer 2005;103(4):821-829

3 Huizinga JD, Thuneberg L, Klüppel M, Malysz J, Mikkelsen $\mathrm{HB}$, Bernstein A. W/kit gene required for interstitial cells of Cajal and for intestinal pacemaker activity. Nature 1995;373(6512):347-349

4 Miettinen M, Sobin LH, Lasota J. Gastrointestinal stromal tumors of the stomach: a clinicopathologic, immunohistochemical, and molecular genetic study of 1765 cases with long-term follow-up. Am J Surg Pathol 2005;29(1):52-68

5 Pierie JP, Choudry U, Muzikansky A, Yeap BY, Souba WW, Ott MJ. The effect of surgery and grade on outcome of gastrointestinal stromal tumors. Arch Surg 2001;136(4):383-389

6 DeMatteo RP, Lewis JJ, Leung D, Mudan SS, Woodruff JM, Brennan MF. Two hundred gastrointestinal stromal tumors: recurrence patterns and prognostic factors for survival. Ann Surg 2000;231(1):51-58

7 FranquemontDW.Differentiation and riskassessment of gastrointestinal stromal tumors. Am J Clin Pathol 1995;103(1):41-47

8 Nilsson B, Sjölund K, Kindblom LG, et al. Adjuvant imatinib treatment improves recurrence-free survival in patients with high-risk gastrointestinal stromal tumours (GIST. Br J Cancer 2007;96(11):1656-1658

9 Iesalnieks I, Rümmele P, Dietmaier W, et al. Factors associated with disease progression in patients with gastrointestinal stromal tumors in the pre-imatinib era. Am J Clin Pathol 2005;124(5):740-748

10 Goss GA, Merriam P, Manola J, Singer S, Fletcher CD, Demetri GD. Clinical and pathological characteristics of gastrointestinal stromal tumors (GIST. Prog Proc Am Soc Clin Oncol 2000;19:599

11 Yang HK, Park DJ, Lee HJ, Kim HH, Kim WH, Lee KU. Clinicopathologic characteristics of gastrointestinal stromal tumor of the stomach. Hepatogastroenterology 2008;55(86-87):1925-1930

12 Fletcher CD, Berman JJ, Corless C, et al. Diagnosis of gastrointestinal stromal tumors: a consensus approach. Hum Pathol 2002;33(5):459-465

13 Kim IH, Kim IH, Kwak SG, Kim SW, Chae HD. Gastrointestinal stromal tumors (GISTs) of the stomach: a multicenter, retrospective study of curatively resected gastric GISTs. Ann Surg Treat Res 2014;87(6):298-303

14 Wang M, Xu J, Zhang Y, et al. Gastrointestinal stromal tumor: 15-years' experience in a single center. BMC Surg 2014;14:93

15 El-Zohairy M, Khalil SA, Fakhr I. El-Shahawy M, Gouda I. Gastrointestinal stromal tumor (GIST)'s surgical treatment, NCI experience. J Egypt Natl Canc Inst 2005;17(2):56-66

16 Call J, Walentas CD, Eickhoff JC, Scherzer N. Survival of gastrointestinal stromal tumor patients in the imatinib era: life raft group observational registry. BMC Cancer 2012;12:90 
17 Hong L, Zhang T, Lin Y, et al. Prognostic analysis of duodenal gastrointestinal stromal tumors. Gastroenterol Res Pract 2018;2018:4812703

18 Tran T, Davila JA, El-Serag HB. The epidemiology of malignant gastrointestinal stromal tumors: an analysis of 1,458 cases from 1992 to 2000. Am J Gastroenterol 2005;100(1):162-168

19 Miettinen M, Lasota J. Gastrointestinal stromal tumors: review on morphology, molecular pathology, prognosis, and differential diagnosis. Arch Pathol Lab Med 2006;130(10):1466-1478

20 Alvarado-Cabrero I, Vázquez G, Sierra Santiesteban FI, Hernández-Hernández DM, Pompa AZ. Clinicopathologic study of 275 cases of gastrointestinal stromal tumors: the experience at 3 large medical centers in Mexico. Ann Diagn Pathol 2007;11(1):39-45

21 Mucciarini C, Rossi G, Bertolini F, et al. Incidence and clinicopathologic features of gastrointestinal stromal tumors. A population-based study. BMC Cancer 2007;7:230

22 Cho MY, Sohn JH, Kim JM, et al. Current trends in the epidemiological and pathological characteristics of gastrointestinal stromal tumors in Korea, 2003-2004. J Korean Med Sci 2010;25(6):853-862

$23 \mathrm{Kim} \mathrm{KH}$, Kim MC, Jung GJ, Kim SJ, Jang JS, Kwon HC. Long term survival results for gastric GIST: is laparoscopic surgery for large gastric GIST feasible? World J Surg Oncol 2012;10:230
24 Lin SC, Huang MJ, Zeng CY, Wang TI, Liu ZL, Shiay RK. Clinical manifestations and prognostic factors in patients with gastrointestinal stromal tumors. World J Gastroenterol 2003;9(12):2809-2812

25 Huang Y, Zhao R, Cui Y, et al. Effect of gastrointestinal bleeding on gastrointestinal stromal tumor patients: a retrospective cohort study. Med Sci Monit 2018;24:363-369

26 Jumniensuk C, Charoenpitakchai M. Gastrointestinal stromal tumor: clinicopathological characteristics and pathologic prognostic analysis. World J Surg Oncol 2018;16(1):231

27 Kim MC, Yook JH, Yang HK, et al. Long-term surgical outcome of 1057 gastric GISTs according to 7th UICC/AJCC TNM system: multicenter observational study from Korea and Japan. Medicine (Baltimore 2015;94(41):e1526

28 Park CH, Kim GH, Lee BE, et al. Two staging systems for gastrointestinal stromal tumors in the stomach: which is better? BMC Gastroenterol 2017;17(1):141

29 Wang $\mathrm{H}$, Chen P, Liu XX, et al. Prognostic impact of gastrointestinal bleeding and expression of PTEN and Ki-67 on primary gastrointestinal stromal tumors. World J Surg Oncol 2014;12:89

30 Dematteo RP, Gold JS, Saran L, et al. Tumor mitotic rate, size, and location independently predict recurrence after resection of primary gastrointestinal stromal tumor (GIST. Cancer 2008;112(3):608-615 